Review Articles

\title{
Immunology of Septic Shock and Traumatic Injury: A Review
}

\author{
${ }^{1}$ William Heuser, ${ }^{2,3}$ Marianne Frieri, ${ }^{4}$ Krishan Kumar and ${ }^{4}$ Anthony Boutin \\ ${ }^{I}$ Department of Pharmacy, St John's University, Queens, New York, USA \\ ${ }^{2}$ Department of Medicine, Nassau University Medical Center, East Meadow, New York, USA \\ ${ }^{3}$ Divison of Allergy and Immunology, Nassau University Medical Center, East Meadow, New York, USA \\ ${ }^{4}$ Department of Emergency Medicine, Nassau University Medical Center, New York, USA
}

Article history

Received: 09-10-2015

Revised: $10-12-2015$

Accepted: 28-12-2015

Corresponding Author:

William Heuser

Department of Pharmacy, St

John's University, Queens,

New York

Email: fightnirish9@aol.com

\begin{abstract}
Sepsis remains a major worldwide cause of morbidity and mortality and remains amongst the leading causes of death in medical Intensive Care Units (ICUs). Increasing injury severity associated with trauma is a significant independent risk factor for sepsis and many of these patients require intensive care unit resource utilization with increased rates of mortality. There are many postulated theories regarding the mechanism surrounding the correlation between trauma and shock. One such theory evaluated the deterioration of the immune system after trauma with the activation of Damage Associated Molecular Proteins (DAMPs) and the potential role of mitochondrial release into the bloodstream following physical injury leading to the onset of the Systemic Inflammatory Response Syndrome (SIRS). Despite these proposed theories, a breach of cellular integrity seems to unravel a multitude of immunologic responses that in essence account for the deleterious symptoms associated with sepsis. The association between traumatic injury and sepsis is very complex. This review explores the multifaceted immunoinflammatory response and gives an in depth immunologic explanation of the fundamental mediators in the initiation and continuation of the inflammatory response following the loss of cellular integrity.
\end{abstract}

Keywords: Sepsis, Trauma, Injury, SIRS, Procalcitonin

\section{Introduction}

\section{Background}

Post-traumatic sepsis is a significant cause of mortality and there are many factors, both demographically including age and race and injury factors including the mechanism and severity of injury that predict post traumatic sepsis to a considerable extent (Osborn et al., 2004). Worldwide, in the general population under the age of 45 , trauma is one of the main causes of death (Wafaisade et al., 2011). Although there are many immediate effects of trauma following the initial injury including cellular hypoxia and/or severe hypovolemic shock secondary to bleeding, traumatic injury leads to an immunologic deterioration in those individuals that survive. All severe posttraumatic complications including SIRS, Acute Respiratory Distress Syndrome (ARDs) and Multiple Organ Dysfunction (MODS) are directly related to synthesis and release of inflammatory mediators into the bloodstream. Trauma can induce immune function changes known as Systemic Inflammation Response Syndrome (SIRS) and immunosuppression as Compensatory Anti-inflammatory Response Syndrome (CARS). SIRS with a known or predicted source of infection is referred to as sepsis. Due to the fact that it is difficult to isolate the microbial inoculums the differential diagnosis between SIRS and sepsis is unclear to many health care professionals. Clinically it is difficult to isolate the microbial inoculums thus making the differential diagnosis between SIRS and sepsis difficult. In multiple trauma situations, both syndromes can develop simultaneously, recently described as Mixed Antagonist Response Syndrome (MARS) (Novotny, 2010). A recent review covered sepsis and the systemic immune response, the role of cytokines and Procalcitonin (PCT), role of steroids in sepsis, innate immunity and reactive oxygen species related to sepsis and the impact on neutrophil function. PCT has been proven to be a superior biomarker for sepsis diagnosis and for the prediction of its clinical outcome. In addition to the standard clinical workup of a patient, including 
history, physical exam and laboratory findings, the use of PCT has many clinical implications in sepsis that can help streamline the potential diagnosis and rule out other differential diagnoses. It is clear that there are several benefits of integrating the use of PCT into practice in the early golden hours of sepsis diagnosis and can undeniably help prescribers treat the patient both more appropriately and efficiently (Frieri et al., 2015a). Although there are many foreseen benefits of PCT integration into the clinical practice there is still a need for better understanding for the role of PCT and its correlation to the immune response. Further clinical trials that explor immunological role of PCT in sepsis are needed to confirm its sensitivity and sensitivity in the sepsis clinical picture.

The body's first line of defense, the epithelial barrier, is often disrupted in trauma allowing for the penetration of microorganisms into the blood stream and a prolonged state of immune-inflammatory dysregulation. SIRS which is manifested by an excessive proinflammatory response due to the immediate blunt trauma is initiated by the innate immune response. This is then followed by a compensatory anti-inflammatory response syndrome which essentially suppresses the adaptive immune response allowing for bacteria to thrive and infiltrate the host's immune system (Thornhill et al., 2010). The combination of a dampened immune response and a hyperinflammatory state mediated by traumatic injury significantly increases the risk for developing acquired infectious complications.

\section{The Role of the Immune System Following Traumatic Shock}

\section{The Role of the Mitochondrion in Trauma and Shock-A Non Infectious Cause of SIRS?}

As "power plants" of eukaryotic cells, providing energy in the form high energy phosphate bonds of ATP, mitochondria are theorized to be from bacterial origin and exist in eukaryotic cells in an endosymbiotic relationship. Due to their close resemblance to the bacteria from which they originated mitochondria appear to elicit a sepsis-like response characteristic of releasing Damage-Associated Molecular Patterns (DAMPS) (BIDMC, 2010). With direct insult from traumatic injury, SIRS causes many of the vital organs to become dysfunctional as a direct consequence of inflammation. The innate immune response that is present from birth remains the focal point that underlie both SIRS and sepsis, activating Pathogen Associated Molecular Patterns (PAMPs) (BIDMC, 2010). These PAMPs activate a group of cellular responses jointly known as the 'Danger Response' that is present in both sepsis and traumatic injury. Trauma associated with blunt force can result in the death of considerable amounts of tissue activating a very similar immune response that is seen with the body's response to external pathogens such as bacteria or viruses. In the presence of trauma normally intra cellular molecules, like mitochondria, can activate Pattern Recognition Receptor molecules (PRR), at which point they become DAMPs. A recent paper published by Watts hypothesized that DAMPs and PAMPs have many similar mechanisms for triggering inflammation in both the setting of trauma and infection and, mitochondria may in fact be ultimately responsible for inflammation in both cases (Watts and Kline, 2003). Due to mitochondria's autonomous functional capacity and their own DNA, which is a completely separate and distinct entity from the genetic material contained within the cell's nucleus, mitochondria in fact share many similarities with bacteria. Both bacteria and mitochondria share similarities in their method of reproduction, the molecular nature of their DNA and their synthesis of n-formylated proteins (BIDMC, 2010; Watts and Kline, 2003). Based on the similarity between mitochondria and bacteria it is plausible to hypothesize that mitochondria can induce a pro inflammatory state with the release of DAMPs into the circulatory system, in the same way that infections do when they release PAMPs following mechanical trauma. Following traumatic or physical injury mitochondria are released into the bloodstream and the fact that they resemble many of the features of bacteria mentioned previously, mitochondria can elicit an immune response very similarly to the way bacteria elicit an immune response in sepsis. Mitochondria which spill into the bloodstream following a physical injury look enough like the bacteria they originated from to elicit an immune response and mirror that of bacterial sepsis. The fact that tissue death caused by traumatic injury has just as much potential for causing SIRS as does infection, treatment modalities aimed at blunting the hyperinflammatory state may arise in the near future with continued research. The plasma membrane, endoplasmic reticulum, Golgi apparatus and the mitochondria are responsible for many of the exceedingly regulated redox reactions of aerobic metabolism and of equal importance are quinones which essentially are electron and proton carriers that play a primary role in the aerobic metabolism of practically every cell in nature. Although these redox reactions produce a significant amount of the energy the body needs to sustain life, these electron transfer reactions also consequently produce Reactive Oxygen Species (ROS) that play important roles in damage to the body at a molecular level (Madeo et al., 2013). Review of the literature, revealed evidence of significant correlations between production of ROS and inflammatory responses in an exponential relationship, as the 
production of ROS increases the greater the inflammatory response is. The underlying mechanisms by which the inflammatory response and ROS production occur was also be discussed (Madeo et al., 2013; Saad et al., 2013).

The role of mitochondria in initiating apoptosis and activating danger signals is well known in the literature. With blunt trauma and tissue cell injury mitochondria become hyperpolarized and the transmembrane potential becomes uncoupled and irreversibly damaged and Reactive Oxygen Species (ROS) production significantly increases as a result of diminishing ATP production, leading inevitably toward irreversible cellular damage via apoptotic mechanisms (Watts and Kline, 2003). The transient opening of pores during this cellular injury leads to the development of mitochondrial component proteins into the cytoplasmic space activating a vicious cycle of danger signals as depicted in Fig. 1.

Mitochondria also produce ROS as a result of cellular hypoxia which effectively induces oxidative damage on both the mitochondrial DNA and proteins residing in the mitochondria. Under normal physiologic conditions our body is able to effectively scavenge these ROS via endogenous antioxidants, however, under conditions of oxidative stress as seen in trauma, the host defensive mechanisms become overwhelmed as a result of the increasing ROS levels and irreversible mitochondrial damage ensues, consequently leading to organelle dysfunction and ultimately cell death (BIDMC, 2010). It is clear that mitochondria play a major role in the physiological response to trauma and shock and often mitochondrial function becomes depressed and eventually dysfunctional with severe insult. As tissue injury generates a state of hypoxia, deranged metabolism of the mitochondria can lead to significant ROS production, which can potentially to damage cellular integrity including cellular protein production and damage to the host host's genomic DNA.

\section{Deterioration of the Immune System after Trauma}

The Injury Severity Score (ISS) and the Acute Physiology And Chronic Health Evaluation (APACHE II) scores help stratify patients based on the severity and extent of traumatic injuries (Flohe et al., 2008). The ISS and APACHE are widely used and remain the gold standard for prediction of multi-organ failure and development of septic complications. As a general statement, patients with higher scores in both of the ISS and APACHE have a higher mortality rate due to multiorgan failure and statistically develop higher rates of septic complications (Flohe et al., 2008). Hyperinflammation following traumatic injury mediates remote end organ damage and may lead to multi-organ failure; In addition immunosuppression secondary to insult of the epithelial barrier promotes an increased risk to acquire infectious complications.

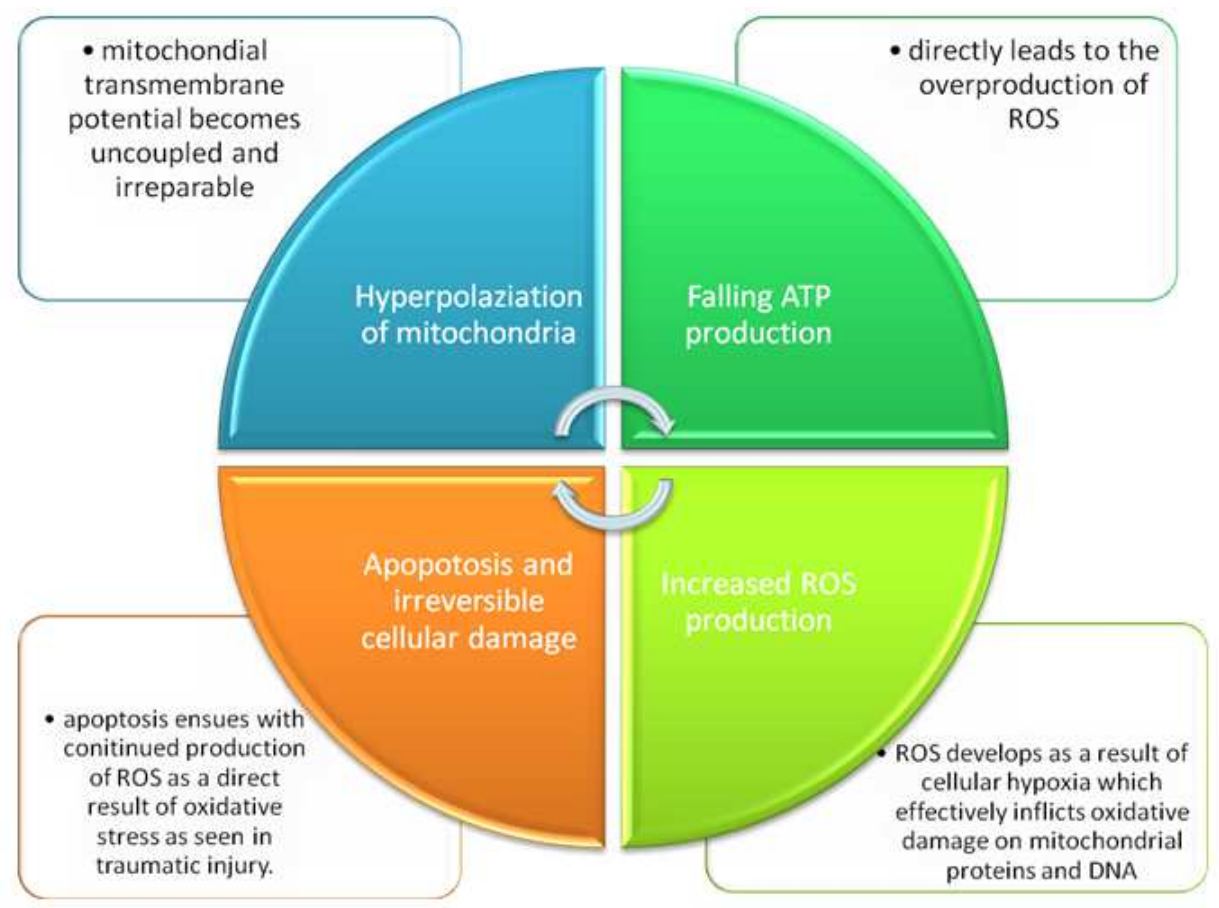

Fig. 1. Vicious cycle of danger signals as a result of leaking mitochondrial components 
How in fact these mechanisms of hyperinflammation and immunosuppression develop are poorly understood however there is developing evidence that damaged tissues which release endogenous danger signals play a developing role in the immune dysfunction seen in traumatic injury (Marshall, 1997). Shock associated with trauma is often associated with major bleeding leading to hypovolemia however the trauma induced disturbance in the host's immune system cannot be solely reduced to the response of hypovolemic shock. A secondary mechanism of autonomic and pathologic dysfunction must account for the trauma-induced modulation of the immune system. Many animal studies have been performed that have aimed to investigate the deterioration of the innate and adaptive immune system following traumatic injury. A study performed by German investigators in 2008 concluded that the initiation of a 'cytokine storm following a hyperinflammatory state in addition to other immunologic mediators including' activation of macrophages and granulocytes results and macrophages result in end organ damage. Also theorized in this study was that downregulation and dysfunction of Antigen-Presenting Cells (APCs) and T-cells, in an immunosupressed state leads to greater risk of attaining infectious complications. Ultimately endogenous danger signals released following traumatic injury were hypothesized to be subsequently involved in both processes (Flohe et al., 2008; Marshall, 1997; Cavaillon and Annane, 2006).

Severe trauma, irrespective of mechanism, commonly causes substantial amounts of blood loss leading to decreased endothelial derived Nitric Oxide (NO), which further leads to augmented platelet aggregation, increased neutrophil infiltration and deregulation of vasorelaxation (Surbatovic et al., 2013). As expected with trauma, loss of endothelial integrity increases vascular permeability, characteristic of the innate immune response reacting to DAMPs. Adaptive immune response is not seen in the initial reaction to trauma as antigen-specific reaction requires more time (Lenz et al., 2007). Antigen presenting cells, such as monocytes and macrophages, effectively recognize the foreign invading pathogen and through uptake into the cytoplasm and lysosome often times are able to kill the microorganism. In septic complications following severe trauma often times monocyte function is impaired further rendering the antigenpresenting capabilities ineffective. Nitric Oxide (NO) and Tumor Necrosis Factor Alpha (TNF-alpha), a pleiotropic inflammatory cytokine, are activated upon epithelial injury which stimulate pro-apototic signaling proteins of immune effector cells, which may contribute to sepsis-associated MODS after severe trauma (Lenz et al., 2007). In addition downstream activation of neurohormal mechanism of catecholamine's and glucocorticoids binding to their corresponding receptors on immune cells also suppress cytokine production and thus impair a competent immune regulatory cell-cell interaction (Tschoeke and Ertel, 2007).

Site targeted complement inhibition is a new pathophysiological pattern that looks at both the beneficial and detrimental role of complement following trauma (Neher et al., 2011). A review on complement considered some basic aspects of complement biology, addressed the clinical effects of hereditary compliment deficiencies as well as the role of compliment related to host cell entry, pathogenesis of infectious diseases and apoptosis. This immunomodulation of autoimmune and inflammatory disorders related to complement components, mechanisms of autoimmune urticaria and tolerance was also discussed (Frieri, 2002). Complement plays a major role in sepsis mediating the activation of the innate immune response however this activation can also contribute to the pathogenesis of trauma induced sequelae and adverse outcome. Complement is able to effectively eliminate pathogens and respond to danger signals exerting its beneficial effect; despite this seeming advantageous effect, complement activation may exert detrimental effects following traumatic injury on host tissue. By mounting an immune response on "innocent" tissue, complement activation is causing more harm than good and damaging viable tissue by exacerbating local and systemic inflammation, thus adding to the antigenic load. The combination of hyperinflammation and exacerbation of toxic mediators consequently sustain SIRS after major trauma and ultimately contributing to remote organ injury and death (Morris et al., 2013).

Endogenous signals such as HMGB-1 and Heat Shock Proteins (HSPs) upon binding to their corresponding receptors (i.e., TLR4) activate macrophage cytokine release in damaged tissue (McGhan and Jaroszewski, 2012). This cascade essentially mediates trauma-induced immune dysfunction through propagation of the inflammatory response following disruption of cellular integrity. It is clear that there are several factors that contribute to the immune response and end organ damage after trauma. The imbalance between a hyperinflammatory state and attenuated immune response contribute to the septic complications following traumatic injury.

\section{Danger Signals Activating the Immune Response} after Trauma: HMGB-1, IL-1a, IL-33

DAMPs, also known as alarmins, are endogenous danger signals that are released from necrotic or stressed cells that effectively initiate the inflammatory response following trauma. There are 3 main alarmins that are central to the inflammatory response including High 
Mobility Group Box Protein 1 (HMGB -1), interleukin 33 (IL-33) and Interleukin-1 alpha (IL-1a) as depicted in Fig. 2. There are other alarmins including galectins, adenosine and thioredoxin that play a role in signaling downstream inflammatory activation however, HMGB1, IL-33 and IL-1a have unique dual-function mediator properties in the initiation of the immune response after trauma that is most concerning to us as practitioners (Hirsiger et al., 2012). A study was done which compared healthy volunteers to pre-oesophagectomy patients, patients with SIRS and/or sepsis and neutropenic patients with sepsis and looked at levels of Thioredoxin (Trx), macrophage Migration Inhibitory Factor (MIF), IL-6, IL-8, IL-10 and Procalcitonin. As expected, plasma levels of these mediators were significantly more prevalent in patients with SIRS/sepsis. A subset analysis was done that compared the different mediators and suggested a unique correlation between that of Trx and MIF. The investigators found that both Trx and MIF were substantially lower in patients in the group of patients with neutropenia compared with those patients enrolled in the SIRS/sepsis group (Leaver et al., 2010). This relationship is noteworthy and further studies are needed to elucidate the implications of this correlation in septic shock associated with traumatic injury.

Alarmins are pro-inflammatory mediators of the immune system and contribute to the activation of a mixture of immune cells including Mast Cells (MC's) and macrophages during infection and tissue damage (Basu et al., 2000). These activated MCs go on to release pro-inflammatory mediators including histamine and several proteases. Although MCs may play a role in the perpetuation of the immune response, they are able to degrade alarmins via a chymase-specific mechanism that has been suggested to serve as a protective and regulatory role. The $\mathrm{MC}$ chymase specific degradation of alarmin specific Heat Shock Protein (HSP70), HMGB1, IL-33 and biglycan, may limit danger induced inflammation however this is not fully understood (Scaffidi et al., 2002). It is important to understand that alarmins are released during injury and include the High Mobility Group Protein B1 (HMGB1) and cytokines of the IL-1 family, such as IL-1 $\alpha$, IL-18 and IL-33 as mentioned previously (Chen et al., 2007; Yang et al., 2007; Moussion et al., 2008; Schmitz et al., 2005). A recent review how trauma can involve a variety of triggers and mechanistic areas related to the various roles of mast cells in trauma, mediators, cytokines and neuroinflammation that play important roles in allergic and immune disorders was published (Frieri et al., 2015b).

\section{Role of HMGB-1}

Sterile injury, which encompasses hemorrhagic shock and ischemia/reperfusion injury induces the release of many inflammatory cytokines however the focus of this section involves the release of HMGB1, a proinflammatory cytokine released by many cells of the immune system (Tsung et al., 2005). As an inflammatory mediator, HMGB1 exerts pleiotropic proinflammatory effects on various organ systems mainly through its ability to activate phagocytic and endothelial cells that promote tissue repair, chemotaxis, angiogenesis and recruitment and proliferation of stem cells (Wang et al., 2004). The activities of HMGB-1 are all mediated through its interaction with pathogenrecognition receptors and Toll Like Receptors (TLRs), which also recognize products from invading microorganisms. In addition, the concentration of HMGB-1 correlate with the severity of injury and the systemic inflammatory response, acting as an early proinflammatory mediator peaking from 2 to 6 hours following traumatic injury (Klune et al., 2008).

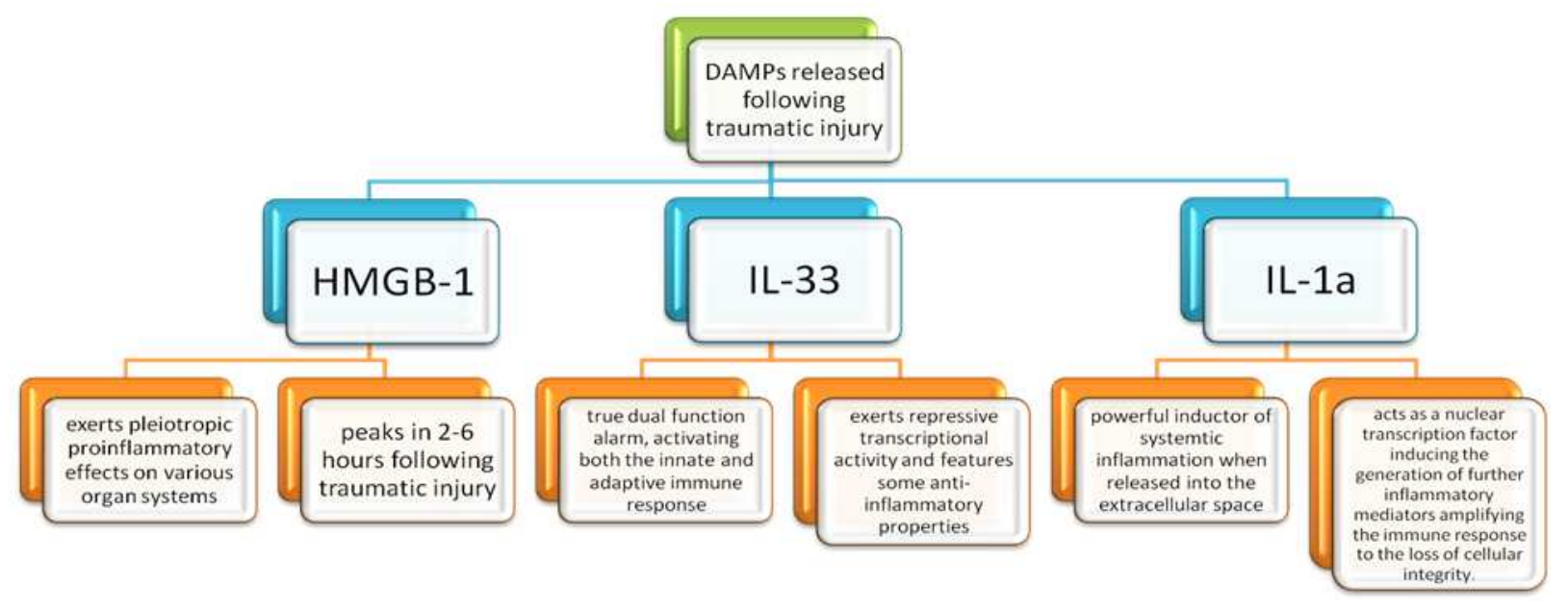

Fig. 2. Danger signals activating the immune response following traumatic injury 


\section{Role of Interleukin-1 $\alpha(I L-1 \alpha)$}

Interleukins play a major role in the immune response; however their role is complex as they have both anti-inflammatory as well as proinflammatory properties. Following the breach of cellular integrity, IL1a along with several other inflammatory cytokines illicit an amplified response characteristic of monocytes and macrophages enhancements of defensive functions in addition to co-stimulation of Natural Killer (NK) cells (Dinarello, 1991). As a dual function alarmin IL1a activates an immune-modulatory response when released by cells of necrotic tissue, aside from its role in intracellular functioning in intact cells as a nuclear transcription factor. IL-1a is rarely intrinsically active and seldom secreted as an active moiety however its precursor molecule (pIL-1a) is biologically active (Dinarello, 1991). IL-1a is only secreted following the loss of cellular integrity and upon activation of monocytic cells, membrane calpain is activated and functions to cleave the precursor (pIL-1a) into the active constituent IL-1a (Wang et al., 2004). Like 1L33 but different in its activity from other members of the interleukin family, IL-1a serves as nuclear transcription factor aside from its role on intracellular and extracellular membrane receptors. As a transcription factor, IL-1a is able to induce downstream signaling to eventually produce NF-k $\beta$ and proinflammatory cytokines such as IL-6 and IL-8 (Werman et al., 2004). A novel mechanism to block the immunomodulatory effects of IL-1a was performed in preclinical studies treating mice with clinically diagnosed SIRS and sepsis with IL-1 receptor antagonists and showed potentially promising results however it was unable to meet the primary endpoint of mortality reduction in a large double-blind, placebo controlled clinical trial (Dinarello, 2001). In summary, IL-1a is distinct from the other interleukins in regards to its role in traumatic injury, serving as a dual function alarmin. Not only does it produce a hyperinflammatory state, characteristic of many of the other interleukins upon release into the extracellular space but uniquely IL-1a serves as nuclear transcription factor which essentially amplifies the immune response through induction of the generation of further inflammatory mediators.

\section{Role of Interleukin-33 (IL-33)}

IL-33 represents the third dual function protein of the alarmin family and very similar in mechanism to HMGB-1 and IL-1a is released following cellular damage. Unlike IL-1a which is cleaved and activated to an active form, it has been elucidated that the fundamental actions of IL-33 is independent of caspase-1-cleavage and in fact, IL-33 may even be potentially inactivated by caspase-1 (Schmitz et al., 2005; Cayrol and Girard, 2008; Talabot-Ayer et al., 2009). As a cytokine and nuclear transcription factor IL-33 fulfills the definition criteria for a dual function alarmin. Unlike HMGB-1 and IL-1a which exert primarily inflammatory properties and increase transcriptional activity, IL-33 does the exact opposite through repressive transcriptional activity and like many of the interleukins, purports a degree of antiinflammatory actions (Kunes et al., 2010). Upon release into the plasma IL-33 is able to activate cells of the innate and adaptive immune response through interaction with specific receptors on the surface of THelper 2 (TH2) cells and mast cells, namely ST2 (Chackerian et al., 2007). Upon interaction with ST2 receptor, IL-33 is able to illicit actions consistent with those of $\mathrm{TH} 2$, including amplified production of cytokines IL-5 and IL-13, both of which have antiinflammatory properties. In addition IL-33 is functions as a chemotactic factor in the mobilization of $\mathrm{TH} 2$ to the site of action and equally as important, induces the differentiation of naïve $\mathrm{T}$ cells towards the lineage of TH2 phenotype production (Kurowska-Stolarska et al., 2008; Komai-Koma et al., 2007). IL-33 has implications in mediating anaphylactic shock as IL-33 is involved in the release of many inflammatory mediators, all of which are hallmark triggers of anaphylactic shock. IL-33 triggers the production and release of proinflammatory cytokines and chemokines such as IL-1 $\beta$, IL-6, or TNF-alpha, promotes maturation and causes degranulation for further inflammatory mediation (De Vos and Frieri, 2015). To date there are no multi-centered trials that explicitly depict the beneficial or detrimental role of IL-33 in trauma however there is indirect evidence of its role in trauma where data has shown that patients who are clinically diagnosed with sepsis or experience a major trauma portray elevated levels of soluble receptors ST2, consistent with the activation of SIRS (Obeid et al., 2007). To sum up the findings of IL-33 it is clear that this dual functioning alarmin activates both the innate and adaptive immune response leading to a multitude of immunologic responses. The actions of IL-33 are heavily dependent on the environment where it exerts either beneficial anti-inflammatory effects or exacerbates inflammatory process' present in traumatic injury.

\section{Conclusion}

The correlation between traumatic injury and sepsis is very complex and the exact immuneinflammatory mechanisms surrounding both processes are not fully understood. Loss of cellular integrity following injury activates the innate response characteristic of cytokine 
storm and a state of hyperinflammation which has implications in remote organ damage and may lead to multi-organ failure. Sepsis, defined by the SIRS criteria, has many overlapping features of those seen in traumatic injury and is essentially indistinguishable from an immunological standpoint. Following severe trauma there is often an imbalance between inflammation and counter-regulation and increasing evidence suggest that damaged tissue plays a role in trauma induced immune dysfunction. The dual function alarmins HMGB-1, IL-1a and IL-33 represent fundamental mediators in both the initiation and continuation of the inflammatory response following the loss of cellular integrity as outlined in this review. The increasing role of mitochondrial which has its origin in bacteria, mimic bacterial infections by activating the immune response after cellular disruption, potentially changing the role of therapeutic interventions in traumatic shock focused on halting danger sensing and transmission of inflammatory mediators. Restorative strategies aimed at targeting DAMPs remain a novel area of research due to their role following tissue injury. The immunologic response to trauma shows great similarity to the systemic inflammatory response elicited in sepsis and treatment modalities should be targeted at treating the underlying cellular dysfunction in the immune response following the loss of cellular integrity. Future studies examining these novel therapeutic mechanisms surrounding traumatic shock are warranted and can offer potentially promising results to clinicians worldwide.

\section{Author's Contributions}

All authors equally contributed in this work.

\section{Compliance with Ethical Requirements}

William Heuser, Marianne Frieri, Krishan Kumar and Anthony Boutin declare that we have no conflict of interest.

\section{References}

Basu, S., R.J. Binder, R. Suto, K.M. Anderson and P.K. Srivastava, 2000. Necrotic but not apoptotic cell death releases heat shock proteins, which deliver a partial maturation signal to dendritic cells and activate the $\mathrm{NF}-\kappa \mathrm{B}$ pathway. Int. Immunol., 12: 1539-1546.

DOI: $10.1093 /$ intimm/12.11.1539

BIDMC, 2010. How trauma leads to inflammatory Response: Mitochondria may be at root $f$ dangerous complications from injury. ScienceDaily, Beth Isreal Deaconess Medical Center.
Cavaillon, J.M. and D. Annane, 2006. Compartmentalization of the inflammatory response in sepsis and SIRS. J. Endotoxin Res., 12: 151-170. PMID: 16719987

Cayrol, C. and J.P. Girard, 2008. The IL-1-like cytokine IL-33 is inactivated after maturation by caspase-1. Proc. Nat. Acad. Sci. USA., 106: 9021-9026. DOI: 10.1073/pnas.0812690106

Chackerian, A.A., E.R. Oldham, E.E. Murphy, J. Schmitz and S. Pflanz et al., 2007. IL-1 Receptor accessory protein and ST2 comprise the IL-33 receptor complex. J. Immunol., 179: 2551-2555. DOI: $10.4049 /$ jimmunol.179.4.2551

Chen, C.J., H. Kono, D. Golenbock, G. Reed and S. Akira et al., 2007. Identification of a key pathway required for the sterile inflammatory response triggered by dying cells. Nat. Med., 13: 851-856. DOI: $10.1038 / \mathrm{nm} 1603$

De Vos, G.S. and M. Frieri, 2015. Anaphylaxis and Mast Cell Disorders. Manual of Allergy and Clinical Immunology for Otolaryngologists.

Dinarello, C.A., 1991. Interleukin-1 and interleukin-1 antagonism. Blood, 77: 1627-1652. PMID: 1826616

Dinarello, C.A., 2001. Anti-cytokine therapies in response to systemic infection. J. Investigative Dermatol. Sympos. Prec., 6: 244-250. DOI: 10.1046/j.0022-202x.2001.00046.x

Flohe, S.B. S. Flohe and F. Schade, 2008. Invited review: Deterioration of the immune system after trauma: Signals and cellular mechanisms. Innate Immunity, 14: 333-344. DOI: $10.1177 / 1753425908100016$

Frieri, M., 2002. Complement-related diseases. Allergy Asthma Proc., 23: 319-324.

Frieri, M., B. Shiferaw, K. Kumar and A. Boutin, 2015a. Role of harmful molecules in the pathogenesis of sepsis: A review. J. Nat. Sci., 1: e142-e142.

Frieri, M., K. Kumar and A. Boutin, 2015b. Role of mast cells in trauma and neuroinflammation in allergy immunology. Ann. Allergy Asthma Immunol., 115: 172-7. DOI: 10.1016/j.anai.2015.06.025

Hirsiger, S., H.P. Simmen, C.M.L. Werner, G.A. Wanner and D. Rittirsch, 2012. Danger signals activating the immune response after trauma. Mediators Inflammat., 2012: 315941- 315950. DOI: $10.1155 / 2012 / 315941$

Klune, J.R., R. Dhupar, J. Cardinal, T.R. Billiar and A. Tsung, 2008. HMGB1: Endogenous danger signaling. Molecular Med., 14: 476-484. DOI: 10.2119/2008-00034.Klune

Komai-Koma, M., D. Xu, Y. Li, A.N.J. McKenzie and I.B. McInnes et al., 2007. IL-33 is a chemoattractant for human Th2 cells. Eur. J. Immunol., 37: 2779-2786. DOI: $10.1002 /$ eji.200737547 
Kunes, P., Z. Holubcova, M. Kolackova and J. Krejsek, 2010. Interleukin-33, a novel member of the IL-1/IL-1 8 cytokine family, in cardiology and cardiac surgery. Thoracic Cardiovascular Surgeon, 58: 443-449.

DOI: $10.1055 / \mathrm{s}-0030-1250436$

Kurowska-Stolarska, M., P. Kewin, G. Murphy, R.C. Russo and B. Stolarski et al., 2008. IL-33 induces antigen-specific IL-5 ${ }^{+} \mathrm{T}$ cells and promotes allergic-induced airway inflammation independent of IL-4. J. Immunol., 181: 4780-4790.

DOI: 10.4049/jimmunol.181.7.4780

Leaver, S.K., N.S. MacCallum, V. Pingle, M.B. Hacking and G.J. Quinlan et al., 2010. Increased plasma thioredoxin levels in patients with sepsis: Positive association with macrophage migration inhibitory factor. Intensive Care Med., 36: 336-41.

DOI: $10.1007 / \mathrm{s} 00134-009-1640-\mathrm{z}$

Lenz, A., G.A. Franklin and W.G. Cheadle, 2007. Systemic inflammation after trauma. Injury, 38: 1336-1345. DOI: 10.1016/j.injury.2007.10.003

Madeo, J., A. Zubair and M. Frieri, 2013. A review on the role of quinones in renal disorders. Springer Plus, 2: 139-139. DOI: 10.1186/2193-1801-2-139

Marshall, J., 1997. Both the disposition and the means of cure: "Severe SIRS," "sterile shock," and the ongoing challenge of description. Crit. Care Med., 25: 1765-1766. PMID: 9366746

McGhan, L.J. and D.E. Jaroszewski, 2012. The role of toll-like receptor-4 in the development of multiorgan failure following traumatic haemorrhagic shock and resuscitation. Injury, 43: 129-136. DOI: 10.1016/j.injury.2011.05.032

Morris, A.C., A.J. Simpson and T.S Walsh, 2013. Hyperinflammation and Mediators of Immune Suppression in Critical Illness. In: Annual Update in Intensive Care and Emergency Medicine, Vincent, J.L. (Ed.), Springer, New York, ISBN-10: 3642351093, pp: 135-144.

Moussion, C., N. Ortega and J.P. Girard, 2008. The IL1-like cytokine IL-33 is constitutively expressed in the nucleus of endothelial cells and epithelial cells in vivo: A novel 'alarmin'? PLoS One, 3: e3331-e3331. DOI: 10.1371/journal.pone.0003331

Neher, M.D., S. Weckbach, M.A. Flierl, M.S. Huber-Lang and P.F. Stahel, 2011. Molecular mechanisms of inflammation and tissue injury after major trauma-is complement the "bad guy"? J. Biomed. Sci., 18: 90-90. DOI: 10.1186/1423-0127-18-90

Novotny, A.R., 2010. Biomarkers in SIRS and sepsis: Quo Vadis? J. Emerg. Trauma Shock, 3: 36-8. DOI: $10.4103 / 0974-2700.58665$
Obeid, M., A. Tesniere, F. Ghiringhelli, G.M. Fimia and L. Apetoh et al., 2007. Calreticulin exposure dictates the immunogenicity of cancer cell death. Nat. Med., 13: 54-61. DOI: 10.1038/nm1523

Osborn, T., J.K. Tracy, J.R. Dunne, M. Pasquale and L.M. Napolitano, 2004. Epidemiology of Sepsis in patients with traumatic injury. Crit. Care Med., 32: 2234-2240. PMID: 15640635

Saad, S., A. Zubair and M. Frieri, 2013. Immune response to nanomaterials: Implications for medicine and literature review. Curr. Allergy Asthma Rep., 13: 50-7. DOI: $10.1007 / \mathrm{s} 11882-012-0302-3$

Scaffidi, P., T. Misteli and M.E. Bianchi, 2002. Release of chromatin protein HMGB1 by necrotic cells triggers inflammation. Nature, 418: 191-195.

DOI: 10.1038 /nature00858

Schmitz, J., A. Owyang, E. Oldham, Y. Song and E. Murphy et al., 2005. IL-33, an interleukin-1-like cytokine that signals via the IL-1 receptor-related protein ST2 and induces T helper type 2-associated cytokines. Immunity, 23: 479-490.

DOI: 10.1016/j.immuni.2005.09.015

Surbatovic, M., M. Veljovic, J. Jevdjic, N. Popovic and D. Djordjevic et al., 2013. Immunoinflammatory response in critically Ill patients: Severe sepsis and/or trauma. Mediators Inflammat., 2013: 362793-362793. DOI: 10.1155/2013/362793

Talabot-Ayer, D., C. Lamacchia, C. Gabay and G. Palmer, 2009. Interleukin-33 is biologically active independently of caspase-1 cleavage. J. Biol. Chem., 284: 19420-19426. DOI: 10.1074/jbc.M901744200

Thornhill, R., D. Strong, S. Vasanth and I. Mackenzie, 2010. Trauma sepsis. Trauma, 12: 31-49. DOI: $10.1177 / 1460408609356828$

Tschoeke, S.K. and W. Ertel, 2007. Immunoparalysis after multiple trauma. Injury. 38: 1365-1372. DOI: 10.1016/j.injury.2007.08.041

Tsung, A., R. Sahai, H. Tanaka, A. Nakao and M.P. Fink et al., 2005. The nuclear factor HMGB1 mediates hepatic injury after murine liver ischemiareperfusion. J. Exp. Med., 201: 1135-1143. DOI: $10.1084 /$ jem.20042614

Wafaisade, A., R. Lefering, B. Bouillon, S.G. Sakka and O.C. Thamm et al., 2011. Epidemiology and risk factors of sepsis after multiple trauma: an analysis of 29,829 patients from the Trauma Registry of the German Society for Trauma Surgery. Crit. Care Med., 39: 621-628. PMID: 21242798

Wang, H., H. Yang and K.J. Tracey, 2004. Extracellular role of HMGB1 in inflammation and sepsis. J. Internal Med., 225: 320-331. DOI: $10.1111 /$ j.1365-2796.2003.01302.x 
Watts, J.A. and J.A. Kline, 2003. Bench to bedside: The role of mitochondrial medicine in the pathogenesis and treatment of cellular injury. Acad. Emerg. Med., 10: 985-997. PMID: 12957984

Werman, A., R. Werman-Venkert, R. White, J.K. Lee and B. Werman et al., 2004. The precursor form of IL-1alpha is an intracrine proinflammatory activator of transcription. Proc. Nat. Acad. Sci. USA., 101: 2434-2439. PMID: 14983027
Yang, D., Q. Chen, H. Yang, K.J. Tracey and M. Bustin et al., 2007. High Mobility group box-1 protein induces the migration and activation of human dendritic cells and acts as an alarmin. J. Leukoc. Biol., 81: 59-66. DOI: 10.1189/jlb.0306180 